\title{
Serum Nesfatin-1 in patients with type 2 diabetes mellitus: A cross sectional study
}

\begin{tabular}{cc}
\hline Niyan Jassim Mohammad $^{1 *}$ & Dler Qader Gallaly $^{1}$ \\
\hline Abstract &
\end{tabular}

Background and objective: Nesfatin-1 is a newly described peptide, derived from nucleobindin2. Nesfatin-1 suppresses food intake and it is involved in regulating insulin secretion. The aim of this study was to compare serum levels of Nesfatin-1 in patients having type 2 diabetes and non-diabetic subjects.

Methods: This cross-sectional study included 90 participants; 64 patients with type 2 diabetes mellitus (32 males and 32 females) and 26 control subjects ( 13 males and 13 female). Body mass index, fasting serum level of glucose, fasting serum level of insulin, and glycated hemoglobin were estimated. Homeostasis model assessment of insulin resistance was calculated. Nesfatin-1 level was measured using enzyme-linked immunesorbent assay kit. The data was analyzed using Graphpad prism 7.04 for windows.

Results: Type 2 diabetes patients aged from 33-78 years and the control group aged from 32-75 years. Nesfatin-1 level in the diabetic group was significantly lower than controls. The median interquartile range (IQR) of Nesfatin-1 was 0.765 (0.4-1.173) in diabetes and 1.02 (0.775-1.458) in controls. The diabetes group has significantly higher homeostasis model assessment of insulin resistance compared with non-diabetics. Serum Nesfatin-1 was correlated negatively with body mass index, fasting serum glucose, fasting serum insulin, glycatedhemoglobin, and homeostasis assessment of insulin resistance.

Conclusion: Serum Nesfatin-1 level is negatively correlated with fasting serum glucose, fasting serum insulin, and glycated hemoglobin. This association supports the role of Nesfatin-1 in increased insulin resistance in patients with type 2 diabetes.

Keywords: Type 2 diabetes mellitus; Nesfatin-1; Homeostasis model assessment of insulin resistance; Glycated hemoglobin.

\section{Introduction}

Nesfatin-1 (NES-1) is a newly discovered multi-functional peptide hormone with an approximate molecular weight of 9.8 kilo Dalton and a half-life of 23.5 minutes. It was first discovered in 2006 by Oh-I and his coworkers. ${ }^{1-3}$ NES-1 is derived from nucleobindin2 (NUCB2) precursor, which is DNA and calcium binding protein that is found in the plasma membrane and neuroplasma. NUCB2 is highly conserved in humans, rats, and mice, that shares more than $85 \%$ of homology between humans and the other mammal species and even demonstrates similarities with lower organisms. ${ }^{3-5}$ The NUCB2 precursor protein is possibly posttranslationally cleaved by the enzyme prohormone-convertase into the $\mathrm{N}$-terminal NES-1amino acids 1-82 (AA 1-82), NES-2 (AA 85-163) and the C-terminal NES-3 (AA 166-396). ${ }^{1,6}$ Several biological actions for NES-1 have been identified, specifically of the middle part of it, which corresponds to AA 24-53, it has a key role in physiological effects of NES-1, particularly for an anorexic effect, whereas no biological action has been described for NES-2 and NES-3. ${ }^{6,7}$ NUCB2 and NES-1 are expressed by the central nervous system (CNS) and peripheral tissues. In CNS, expressed prominently in the

${ }^{1}$ Department of Medical Physiology, College of Medicine, Hawler Medical University, Erbil, Iraq.

* Correspondence: niyan.jassim@hmu.edu.krd 
Hypothalamus, particularly in the supraoptic and paraventricular nuclei, spinal cord autonomic nuclei, pituitary gland, brain stem nuclei, nucleus tractus-solitarus, and in peripheral tissues including gastric mucosa, adipocytes, pancreatic endocrine beta cells ( $\beta$-cells), and testis tissue. It has been found that its expression level is 20-fold higher in the gastric oxyntic mucosa than in the brain. ${ }^{8,9}$ It has been reported that NES-1 suppresses food intake and reduces weight gain when injected into the third ventricle of rodents or it administered peripherally. So, the major function of NES-1 is inhibition of food intake in time-dependent, dose-dependent, and insulin-dependent manners. ${ }^{10,11}$ Detection of NES-1 localizes with insulin in the pancreatic beta islets indicates the involvement of NES-1 in regulating insulin secretion from pancreatic beta-cells and thus regulation of blood glucose. NES-1 affects glucose metabolism, the suggested mechanism for that is by increasing insulin sensitivity and decreasing insulin resistance. For measuring insulin resistance, homeostatic model assessment of insulin resistance (HOMA-IR) is used. 5,9,15-17 Type 2 diabetes mellitus (T2DM) is an important expanding global health problem. It is associated with alteration in glucose metabolism, insulin resistance, abnormality in fasting serum glucose (FSG), and also impaired glucose tolerance. ${ }^{18,19}$ Factors such as obesity, unhealthy diet, low physical activity, and genetic factors contribute to some pathophysiological disturbances that are responsible for impaired glucose homeostasis in T2DM. Impaired insulin secretion and insulin resistance are still the core defects in T2DM. ${ }^{20}$ This study aimed to find out the level of serum NES-1 in patients having T2DM and to compare it with a serum level of NES-1 in normal subjects. It also aimed to find out the relation between levels of NES-1 and T2DM and detect the relation between NES-1, insulin level, and insulin resistance.

\section{Methods}

\section{Subjects}

This cross-sectional study was conducted at Layla Qasim Diabetic center in Erbil city in January and February 2018. The study included 90 participants: 64 patients with T2DM and 26 subjects free from diabetes mellitus comprised the control group. The diagnoses of Type 2 DM were made at least before one year by a physician according to WHO criteria. After verbal consent was obtained from the participants, information was obtained from them using a questionnaire designed for the study. Body weight and height were measured in all subjects using a scale (Seca-USA) and a wall-mounted stadiometer to the nearest $0.5 \mathrm{~kg}$ and $0.5 \mathrm{~cm}$, respectively. Body mass index (BMI) was calculated as the body weight in kilograms divided by the height in meters squared. The subjects in the control group had normal glucose levels (no diabetes history). Clinical exclusion criteria for cases and controls were Type 1 DM, pregnancy, hormone replacement therapy, cancer, severe acute or chronic infectious disease, systemic diseases (heart failure, kidney, liver, or lung diseases), thyroid, and adrenal disorders. All procedures were conducted in accordance with The Code of Ethics of the World Medical Association (Declaration of Helsinki) for experiments involving humans. ${ }^{21}$ The Local Ethics Committee at the College of Medicine, Hawler Medical University approved the study protocol.

\section{Biochemical parameters and hormone analyses}

Blood samples $(5 \mathrm{ml})$ were drawn from a forearm vein in the morning after overnight fasting for hormone and other biochemical analyses. About $2.5 \mathrm{ml}$ of the blood sample was placed into vacuum blood collection tube and then centrifuged at $4500 \mathrm{rpm}$ for 5 minutes to obtain serum, some of the separated serum was used to estimate FSG and some for fasting serum insulin ( $\mathrm{FSI}$ ) estimation by using an automated immunoassay analyzer 
(Cobas e411, Roche Diagnostics $\mathrm{GmbH}$, D-68298 Mannheim, Germany), while the rest of the serum was immediately placed in Eppendorf tubes and frozen at $-40{ }^{\circ} \mathrm{C}$ to be used later for the analysis of NES-1. The other $2.5 \mathrm{ml}$ of the blood sample was placed into tubes containing Ethyle Diamine Tetra Acetic Acid (EDTA) for direct analysis of glycated hemoglobin $\left(\mathrm{HbA}_{1 \mathrm{C}}\right)$ using (GesanChem 200, Campobello di Mazara- Italy). The HOMA-IR calculated using the following equation:

HOMA- IR $=\mathrm{FSI}(\mathrm{mlU} / \mathrm{L}) \times \mathrm{FSG}(\mathrm{mg} / \mathrm{dl}) /$ 405. ${ }^{15,16}$

\section{Serum NES-1 assay}

Fasting serum NES-1 level was measured using a commercial enzyme-linked immune -sorbent assay (ELISA) kit (Bioassay Technology Laboratory, YangpuDist, Shanghai, China), by Absorbance Microplate Reader (ELx800 ${ }^{\mathrm{TM}}$, Operator's Manual , Biokit, Wefen com. Spain ) The standard curve range was $0.3-90 \mathrm{ng} / \mathrm{ml}$. The coefficients of variation for inter-assay and the intra-assay were $10 \%$ and $8 \%$, respectively.

\section{Statistical analysis}

The data was analyzed using the Graphpad Prism 7.04 for windows. All data were expressed as mean \pm standard error of the mean (SE) or median IQR. Statistical analysis was used according to the (t-test for two independent samples), and the Mann-Whitney test applied for data, which was not normally distributed. Data accuracy for diagnosis of T2DM was presented in terms of sensitivity and specificity by the Receiver Operating Characteristic (ROC) curve, which is a graphical display of sensitivity on the $y$-axis and (1-specificity) on the x-axis for varying cut-off points of test values. The area under the curve (AUC) (normal range $0.5-1$ ) is used for measuring the quantitative accuracy. An area of 0.5 represents a worthless test, while an area of 1 represents a perfect test. Rough guide for classifying the accuracy of a diagnostic test is the traditional academic point system: $0.90-1=$ excellent, $0.80-0.90=$ good, $0.70-0.80=$ fair, $0.60-0.70=$ poor, $0.50-0.60=$ fail. $^{22}$ Correlations between data variables showed by Spearman correlation coefficient ${ }^{\circledR} .{ }^{23} \mathrm{~A} P$ value of less than 0.05 was considered as statistical significant.

\section{Results}

The study included 90 participants: 64 patients with T2DM (32 males; 32 females) aged from 33 to 78 years and 26 subjects free from DM comprised the control group (13 males; 13 female) aged from 32 to 75 . The mean BMI of T2DM cases and controls were $30.21 \pm 0.692$ and $27.76 \pm 0.952$, respectively.

Comparison of biochemical parameters between T2DM and non-diabetic groups

As shown in Table 1 and Figure 1, the fasting serum NES-1 level was significantly $(P=0.042)$ lower in patients with T2DM in comparison with the control group and the median IQR was $0.765(0.4-1.173) \mathrm{ng} / \mathrm{ml}$ and $1.02(0.775-1.458) \mathrm{ng} / \mathrm{ml}$, respectively. The FSG, FSI, $\mathrm{HbA}_{1 \mathrm{c}}$ and the calculated HOMA-IR levels were significantly higher in patients with T2DM compared to the control group. At the same time, the calculated BMI was also higher in the T2DM group, but the difference was not significant statistically. The results were expressed as Mean \pm SEM, except for serum NES-1, FSI, HOMA-IR levels in which they were expressed as median IQR because their data was not normally distributed (Table 1). 
Table 1: Comparison between the groups studied according to laboratory investigations fasting serum levels of NES-1, glucose and insulin, and calculated HOMA-IR, calculated $\mathrm{BMI}$ and $\mathrm{HbA}_{1 \mathrm{c}}$ between the study groups.

\begin{tabular}{lccc}
\hline Parameters & Control $(\mathbf{n}=\mathbf{2 6})$ & T2DM (n=64) & $P$ values \\
\hline NES-1(ng/ml): Median IQR & $1.02(0.775-1.458)$ & $0.765(0.4-1.173)$ & 0.042 \\
FSI (mIU/L): Median IQR & $11.21(7.768-14.44)$ & $15.44(9.42-24.77)$ & 0.033 \\
HOMA-IR: Median IQR & & & \\
FSG (mg/dl) & $2.39(1.823-3.2)$ & $5.15(2.35-9.875)$ & $<0.001$ \\
BMI (kg/m $\left.{ }^{2}\right)$ & $88.96 \pm 1.659$ & $165.9 \pm 10.42$ & $<0.001$ \\
HbA1C $(\%)$ & $27.76 \pm 0.952$ & $30.21 \pm 0.692$ & 0.051 \\
\hline (IQR)= Interquartile range & & & $<0.001$ \\
\hline
\end{tabular}

$(I Q R)=$ Interquartile range

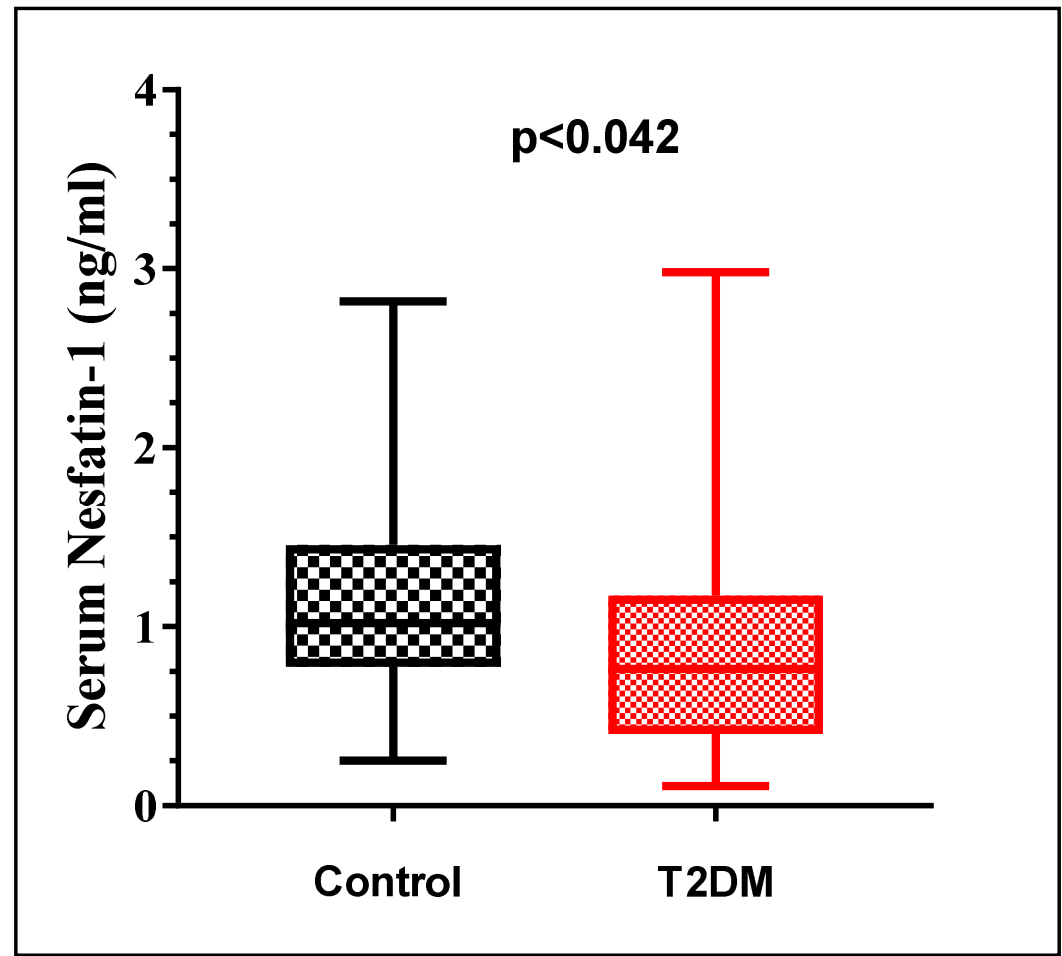

Figure 1: Comparison between serum NES-1 levels in control and T2DM groups. 
Correlation coefficients $(r)$ between data variables

Table 2 shows the correlations between data variables by correlation coefficient $(r)$ and $P$ values. NES- 1 showed no significant negligible negative correlation with $\mathrm{BMI}$, FSG, HbA1C, and FSI. However, it has a significant negligible negative correlation with HOMA-IR (Figure 2). On the other hand, our results showed that BMI had a significant low positive correlation with insulin and HOMA-IR and a significant negligible positive correlation with $\mathrm{HbA}_{1 \mathrm{c}}$. Moreover, the ROC curve of NES-1 shows discrimination between T2DM and control subjects, with an AUC of 0.64 and $P=0.042$, as seen in Figure 3 .

Table 2: Correlations between data variables in all subjects represented by correlation coefficient ( $r$ ) and $P$ values $(p)$ :

\begin{tabular}{lccccccc}
\hline & & NES-1 & BMI & FBG & HbA1C & Insulin & HOMA-IR \\
\hline NES-1 & $\mathrm{R}$ & --------- & -0.011 & -0.216 & -0.07 & -0.05 & -0.221 \\
& $\mathrm{p}$ & --------- & 0.919 & 0.057 & 0.511 & 0.665 & 0.042 \\
BMI & $\mathrm{R}$ & -0.011 & ---1 & 0.188 & 0.214 & 0.42 & 0.416 \\
& $\mathrm{p}$ & 0.919 & ------- & 0.099 & 0.043 & 0.004 & 0.005 \\
FSG & $\mathrm{R}$ & -0.216 & 0.188 & ------ & 0.867 & 0.206 & 0.628 \\
& $\mathrm{p}$ & 0.057 & 0.099 & -------- & $<0.001$ & 0.071 & 0.009 \\
HbA1C & $\mathrm{R}$ & -0.07 & 0.214 & 0.867 & ------- & 0.094 & 0.405 \\
& $\mathrm{p}$ & 0.511 & 0.043 & $<0.001$ & -------- & 0.409 & 0.004 \\
Insulin & $\mathrm{R}$ & -0.05 & 0.42 & 0.206 & 0.094 & -------- & 0.841 \\
& $\mathrm{p}$ & 0.665 & 0.004 & 0.071 & 0.409 & -------- & $<0.001$ \\
\multirow{2}{*}{ HOMA-IR } & $\mathrm{R}$ & -0.221 & 0.416 & 0.628 & 0.405 & 0.841 & -------- \\
& $\mathrm{p}$ & 0.042 & 0.005 & 0.009 & 0.004 & $<0.001$ & -------- \\
\hline
\end{tabular}

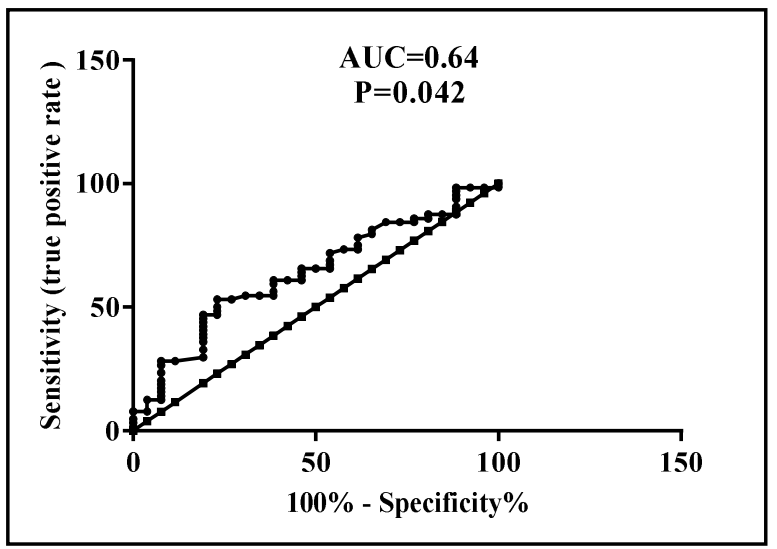

Figure 2: Correlation coefficient between NES-1 and HOMA-IR.

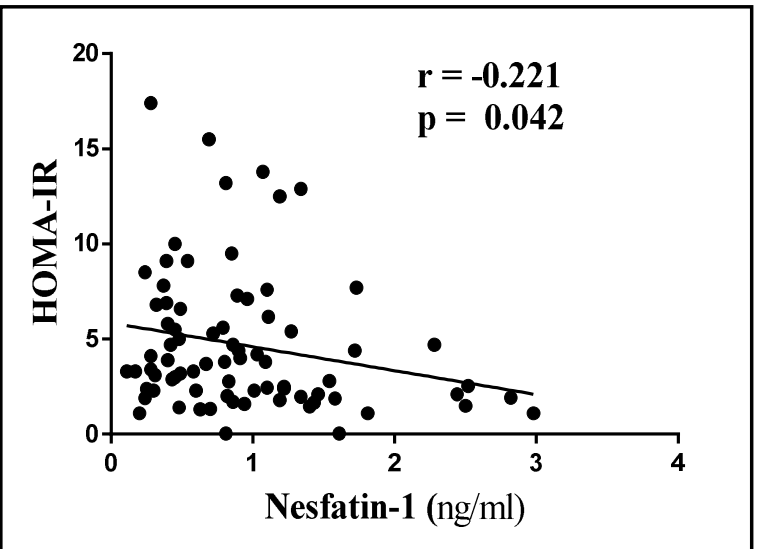

Figure 3: The ROC curve shows the sensitivity and specificity of NES-1 concentration during T2DM. AUC: Area under the curve 


\section{Discussion}

In the present study, we compared fasting serum NES-1 levels in patients having T2DM with non-diabetic persons. We found that NES-1 level was significantly $(P=0.0425)$ lower in patients with T2DM compared to non-diabetic subjects. This result is in agreement with documented results in some previous studies. ${ }^{19,24}$ This finding suggests that low level of serum NES-1 in T2BM patients might be one of the factors causing diabetic hyperphagia because it has been reported that NES-1 has a reducing effect on food intake. Thus, it acts as an anorexigenic agent, but the regulatory mechanism remains not clear. ${ }^{1}$ On the other hand, the ROC curve analysis showed that NES-1 represents as a biomarker for T2DM, with an AUC of 0.64 and $P=0.0042$. The exact mechanism by which NES-1affect T2DM is not fully understood yet, however, some researches support our finding. ${ }^{19,24}$ The results of our study show increased FSG, $\mathrm{HbA}_{1 \mathrm{C}}, \mathrm{FSI}$, and HOMA-IR in T2DM as compared with the non-diabetic group. Calculating of data according to HOMA-IR formula shows that there is a significant difference $(P<0.001)$ in insulin resistance between T2DM and control groups, so; lower NES-1 levels in T2DM group could explain the significantly high insulin resistance in this group because as mentioned before NES-1 suggested to affect glucose metabolism by increasing insulin sensitivity and decreasing insulin resistance. ${ }^{5,9,17}$ Furthermore, our results demonstrated that serum NES-1 level is negatively correlated with all the FSG, $\mathrm{HbA}_{1 \mathrm{C}}, \mathrm{FSI}, \mathrm{HOMA}-\mathrm{IR}$, and BMI. These negative correlations can be explained by the role of NES-1 in glucose metabolism and its anorexigenic effect, and this finding agreed with the results of a study done by Tsuchiya and his coworkers. ${ }^{25}$ An important point to be mentioned is that in our study, the least duration of diagnosis was one year, and newly diagnosed T2DM patients were not included, in which most of them were on oral hypoglycemic drugs. This made the difference with another study done by Zhang and his colleagues (2012) that included newly diagnosed T2DM patients in which the level of NES-1 was higher in T2DM group compared with the control group and the author explained this by the possibility that $\beta$-cells secrete normal amounts of insulin and NES-1 during the early stages of diabetes only. Hence, they designed their study based on excluding effects of microvascular and macrovascular complications and multiple medications on plasma NES-1. ${ }^{4}$ Recent studies reported anti-inflammatory effects for NES-1, and the explained mechanism is that there are elevated circulating NES-1 levels in newly diagnosed T2DM patients, as discovered by Zhang and his coworkers, and this elevation of NES-1 can reduce blood glucose, decrease food intake, increasing lipid metabolism, and countering inflammation. ${ }^{4,26}$ Accordingly, we hypothesized that in newly diagnosed T2DM there will be a compensatory mechanism that leads to the elevation of NES-1 level. In patients with T2DM who are receiving anti-diabetic treatment, circulating NES-1 levels decreased, by the of action of anti-diabetic treatments, as these drugs causing the reduction of blood glucose, increased insulin sensitivity, and additionally controlled food intake. ${ }^{27}$ For the future, we recommend more studies concerning the NES-1 levels in response to diabetic therapy, including the effects of insulin and oral anti-diabetic treatment is needed. To elucidate the physiological role of NES-1, further studies are required. Establishing the physiological and biochemical effects of NES-1 on the regulatory mechanisms of energy systems in the body will contribute to clarify the factors that could play a role in the impairment of glucose metabolism.

\section{Conclusion}

Serum Nesfatin-1 levels are negatively correlated with fasting serum glucose, fasting serum insulin, and glycated hemoglobin. This association supports the 
role of Nesfatin-1 in increased insulin resistance in patients with type 2 diabetes mellitus.

\section{Competing interests}

The authors declare no competing interests.

\section{References}

1. Oh-I S, Shimizu H, Satoh T, Okada S, Adachi S, Inoue $\mathrm{K}$, et al. Identification of nesfatin-1 as a satiety molecule in the hypothalamus. Nature 2006; 443(7112):709-12.

2. Butler AA. More news about NUCB2/Nesfatin-1: A new factor in the hypothalamic control of glucose homeostasis. Diabetes2012; 61(8):1920 2.

3. Aydin S. Role of NUCB2/nesfatin-1 as a possible biomarker. Current Pharmaceutical Design 2013; 19(39):6986-92.

4. Zhang Z, Li L, Yang M, Liu H, Boden G, Yang G. Increased plasma levels of Nesfatin-1 in patients with newly diagnosed type 2 diabetes mellitus. Exp Clin Endocrinol Diabetes 2012; 120(02):91-5.

5. Ayada C, Toru U, Korkut Y. Nesfatin-1 and its effects on different systems. Hippokratia 2015; 19(1):4-10.

6. Stengel A, Mori M, Tache Y. The role of nesfatin-1 in the regulation of food intake and body weight recent developments and future endeavors Andreas. Obes Rev 2013; 14(11):859-70.

7. Prinz P, Teuffel $P$, Lembke V, Kobelt $P$, Goebel-Stengel M, Hofmann $T$, et al. Nesfatin-130-59 injected intracerebroventricularly differentially affects food intake microstructure in rats under normal weight and diet-induced obese conditions. Front Neurosci 2015; 9:422.

8. García-Galiano D, Navarro VM, Gaytan F, Tena-Sempere M. Expanding roles of NUCB2/ nesfatin-1 in neuroendocrine regulation. $\mathrm{J} \mathrm{Mol}$ Endocrinol 2010; 45(5):281-90.

9. Khalili S, Khaniani MS, Afkhami F, Derakhshan MS. NUCB2/Nesfatin-1: A potent meal regulatory hormone and its role in diabetes. EJMHG 2017; 18(2):105-9.

10. Shimizu $H$, Oh-I S, Hashimoto $K$, Nakata M, Yamamoto S, Yoshida N, et al. Peripheral administration of nesfatin-1 reduces food intake in mice: The leptin-independent mechanism. Endocrinology 2009; 150(2):662-71.

11. Stengel A, Goebel M, Wang L, Rivier J, Kobelt $P$, Mönnikes $H$, et al. Central nesfatin-1 reduces dark-phase food intake and gastric emptying in rats: Differential role of corticotropin-releasing factor 2 receptor. Endocrinology 2009; 150 (11):4911-9.

12. Yoshimura M, Matsuura T, Ohkubo J, Maruyama $T$, Ishikura $T$, Hashimoto $H$. A role of nesfatin-1/ NucB2 in dehydration-induced anorexia.
Am J Physiol Regul Integr Comp Physiol 2014; 307(2):225-36.

13. Pan W, Hsuchou H, Kastin AJ. Nesfatin-1 crosses the blood-brain barrier without saturation. Peptides 2007; 28(11):2223-8.

14. Dore R, Levata L, Lehnert $H$, Schulz C. Nesfatin-1: Functions and physiology of a novel regulatory peptide. J Endocrinol 2017; 232(1):45 -65 .

15. Song $\mathrm{Y}$, Manson JE, Tinker L, Howard BV, Kuller LH, Nathan L, et al. Insulin sensitivity and insulin secretion determined by homeostasis model assessment and risk of diabetes in a multiethnic cohort of women: The women's health initiative observational study. Diabetes Care 2007; 30(7):1747-52.

16. Salgado AL, Carvalho LD, Oliveira AC, Santos VN, Vieira JG, Parise ER. Insulin resistance index (HOMA-IR) in the differentiation of patients with non-alcoholic fatty liver disease and healthy individuals. Arq Gastroenterol 2010;47(2):165-9.

17. Li Z, Gao L, Tang H, Yin Y, Xiang X, Li Y, et al. Peripheral effects of Nesfatin-1 on glucose homeostasis. PLoS One 2013; 8(8):e71513.

18. WHO. Definition and diagnosis of diabetes mellitus and intermediate hyperglycemia. Geneva: WHO; 2006.

19. Algul S, Ozkan Y, Ozcelik O. Serum nesfatin-1 levels in patients with different glucose tolerance levels. Physiol Res 2016; 65(6):979-85.

20. DeFronzo RA, Ferrannini E, Groop L, Henry RR, Herman WH, Holst JJ, et al. Type 2 diabetes mellitus. Nat Rev Dis Primers 2015; 1:15019.

21. World Medical Association Declaration of Helsinki. Ethical Principles for Medical Research Involving Human Subjects. Bulletin WHO 2001; 79(4):373-4.

22. $\mathrm{Li} \mathrm{F}, \mathrm{He} \mathrm{H}$. Assessing the accuracy of diagnostic tests. Shanghai Arch Psychiatry 2018; 30(3):207.

23. Hinkle DE, Wiersma W, Jurs SG. Applied Statistics for the Behavioral Sciences. $5^{\text {th }}$ ed. Boston: Houghton Mifflin; 2003.

24. Liu F, Yang Q, Gao N, Liu F, Chen S. Decreased plasma nesfatin-1 level is related to the thyroid dysfunction in patients with type 2 diabetes mellitus. J Diabetes Res 2014; 2014.

25. Tsuchiya T, Shimizu H, Yamada M, Osaki A, Oh-I S, Ariyama $Y$, et al. Fasting concentrations of nesfatin-1 are negatively correlated with body mass index in non-obese males. Clin Endocrinol 2010; 73(4):483-90.

26. Ozturk CC, Oktay S, Yuksel M, Akakin D, Yarat A, Cakir KO. Anti-Inflammatory effects of nesfatin-1 in rats with acetic Acid-Induced colitis and underlying mechanisms. J Physiol Pharmacol 2015; 66(5):741-50.

27. Zhai $\mathrm{T}$, Li SZ, Fan XT, Tian Z, Lu XQ, Dong J. Circulating Nesfatin-1 levels and type 2 diabetes: A systematic review and metaanalysis. J Diabetes Res 2017; 2017:7687098. 